\title{
Rapid implementation of telemedicine in Neurology during the COVID-19 pandemic
}

\section{Challenges in King Abdulaziz Medical City-Jeddah}

Hussein A. Algahtani, MD, FRCPC, MMed, Bader H. Shirah, MD.

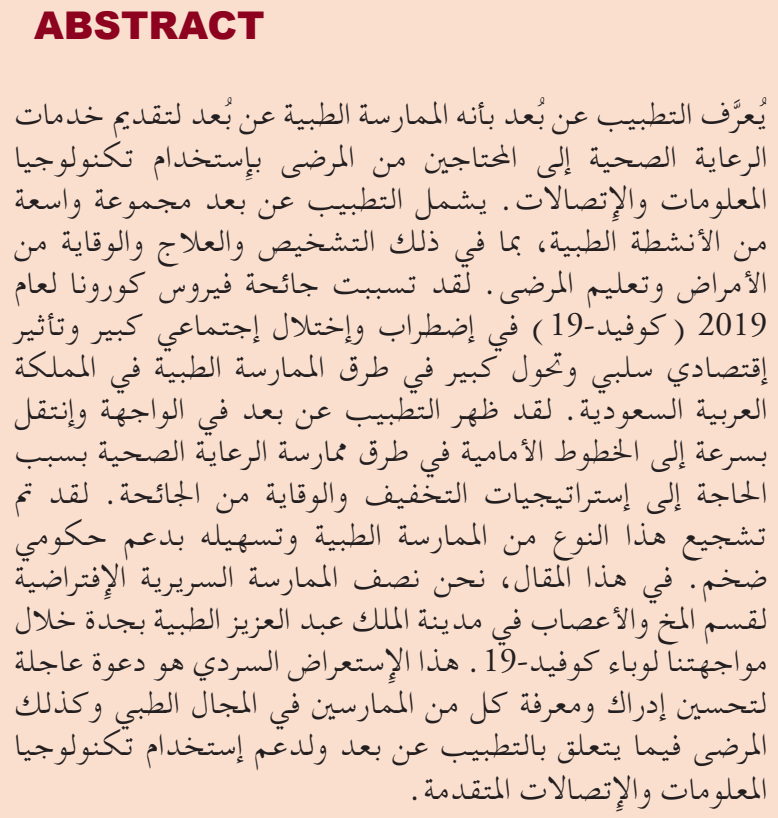

Telemedicine is defined as the remote medical practice of delivering healthcare services to the underserved using information and communication technology. It encompasses a wide range of medical activities, including diagnosis, treatment, disease prevention, and education. The coronavirus disease of 2019 (COVID-19) pandemic has caused significant social dislocation, negative economic impact, and a major change in medical practice in Saudi Arabia. Telemedicine has rapidly moved to the frontline of healthcare practice due to the demand for prevention and mitigation strategies. It has been encouraged and facilitated with huge government support. Herein, we describe the virtual clinical practice of the neurology department at King Abdulaziz Medical City-Jeddah in response to the COVID-19 pandemic. This narrative review is an urgent call to improve the perception and knowledge of both medical personnel and patients concerning telemedicine and to support the utilization of advanced information and communication technology.
Neurosciences 2022; Vol. 27 (1): 4-9

doi: 10.17712/nsj.2022.1.20210080

From the Neurology Section (Algahtani), Department of Medicine, King Abdulaziz Medical City, from King Abdullah International Medical Research Center (Algahtani), from the College of Medicine (Algahtani), King Saud bin Abdulaziz University for Health Sciences, and from Department of Neuroscience (Shirah), King Faisal Specialist Hospital \& Research Centre, Jeddah, Kingdom of Saudi Arabia.

Address correspondence and reprint request to: Dr. Hussein A. Algahtani, Neurology Section, Department of Medicine, King Abdulaziz Medical City, Jeddah, Kingdom of Saudi Arabia. E-mail: halgahtani@hotmail.com

ORCID ID: https://orcid.org/0000-0001-9484-9838

$T$ he World Health Organization defines telemedicine as "The delivery of health care services, where distance is a critical factor, by all health care professional using information and communication technologies for the exchange of valid information for diagnosis, treatment and prevention of disease and injuries, research and evaluation, and for continued education of healthcare providers, all in the interests of advancing the health of individuals and their communities". ${ }^{1}$ The prefix 'tele' is derived from the Greek for 'at a distance'. Hence, telemedicine is defined simply as medicine at a distance or providing medical care to a patient by a physician or other healthcare provider remotely. ${ }^{2}$

In Saudi Arabia and most countries in the world including the United States of America, clinical medicine has been transformed by the coronavirus disease of 2019 (COVID-19) pandemic. ${ }^{3}$ This disease was declared a global pandemic on March 11, 2020. ${ }^{4}$ Due to the rapid spread of the virus, there were more than 549,000 confirmed cases in Saudi Arabia as of November 13, 2021.5 Given the need for strict social isolation, the government implemented a curfew to protect both citizens and expatriates and to limit the 
Table 1 - Major challenges hindering the successful implementation of telemedicine programs and virtual neurology clinics in Saudi Arabia

Some patients live in rural areas with no or limited access to the internet or mobile devices, which makes communication difficult and almost impossible in some cases

Due to cultural reasons, some female patients do not accept virtual neurology clinics and prefer face-to-face in-person clinic visits in the presence of their relatives

Some patients, whether men or women, are not willing to be treated with telemedicine for various personal reasons

Some patients may have cognitive, visual, or hearing impairments, which may hinder direct-to-patient telehealth encounters

Other challenges include a lack of regulations, approved strategies, and plans for implementing telemedicine as well as a lack of availability of adequate experts to operate and maintain telemedicine programs

Some stakeholders may be unwilling to make the required modifications to healthcare processes and bylaws beyond the COVID-19 crisis

Healthcare personnel cannot access electronic medical records from areas outside the hospital for unconvincing reasons such as patient confidentiality. Thus, patient history, laboratory work, and radiology are inaccessible at night during medical consultations, and the patient cannot be interviewed for emergency conditions

Telemedicine is not part of any policy, mission, vision, or needs in most Saudi hospitals. Ensuring the conformity of these aspects with telemedicine is mandatory

Some Saudi hospitals are not equipped with advanced health informatics including electronic medical records, which hinders the application of telemedicine

There are limitations regarding neurological examination and difficulties in performing specific examination maneuvers that require onsite assistance or special equipment

Telemedicine is not part of the undergraduate medical college curriculum or postgraduate residency training programs

spread of this highly infectious disease. ${ }^{6}$ Since then, clinical departments including neurology throughout Saudi Arabia have turned to non-face-to-face visits as a means of providing patient care.

King Abdulaziz Medical City-Jeddah, a 751-bed medical complex, was among the earliest healthcare systems in Saudi Arabia to be directly affected by COVID-19. The medical city was prepared to care for a large number of COVID-19 inpatients while simultaneously providing continuous high standards of clinical excellence for other patients. Neurology outpatient care clinics were transformed from purely traditional in-person, office-based encounters to an almost universal system of direct-to-patient virtual visits. Herein, we describe our experience with the rapid transition to telemedicine and offer recommendations for other important aspects including medical education and potential implementation challenges.

This article is a narrative review of the topic and is considered a call for further research regarding telemedicine in Saudi Arabia. We believe that this narrative review will be of interest to clinicians from different specialties as the COVID-19 pandemic remains a serious public health concern. Further, the application of telemedicine as a sustainable solution to effective and efficient patient care, at a time when social distancing protocols need to be maintained will be of great relevance to the global medical community and society at large.
Telemedicine in Saudi Arabia in response to the COVID-19 pandemic. In response to the COVID-19 pandemic in 2020, medical practices in Saudi Arabia developed and implemented unprecedented changes to healthcare delivery systems. Telemedicine has since rapidly moved to the frontline of clinical practice in Saudi hospitals due to the need for prevention and mitigation strategies. These have been encouraged, facilitated, and enabled by changes in government rules and regulations and huge steps taken by national hospitals to implement them. In almost all Saudi hospitals, all outpatient clinic visits were conducted through virtual means. In addition, all medications were delivered to patients at home using courier delivery services. Hospital beds were only utilized for emergency conditions and infusion therapies that could not be postponed.

Telemedicine in King Abdulaziz Medical CityJeddah. The adoption of smartphones and tablets with broad-band networking capabilities and the presence of highly sophisticated computer-based electronic files have encouraged King Abdulaziz Medical City-Jeddah to provide patient care through virtual neurology clinics using their personal devices at home. These virtual neurology clinics connected the neurology team (using hospital landlines and mobile phones) directly to the patient using the patient's own mobile phone. Simultaneously, the healthcare provider was able to view an electronic file, detailing the patient's medical history, previous clinical examinations, medical reports, 


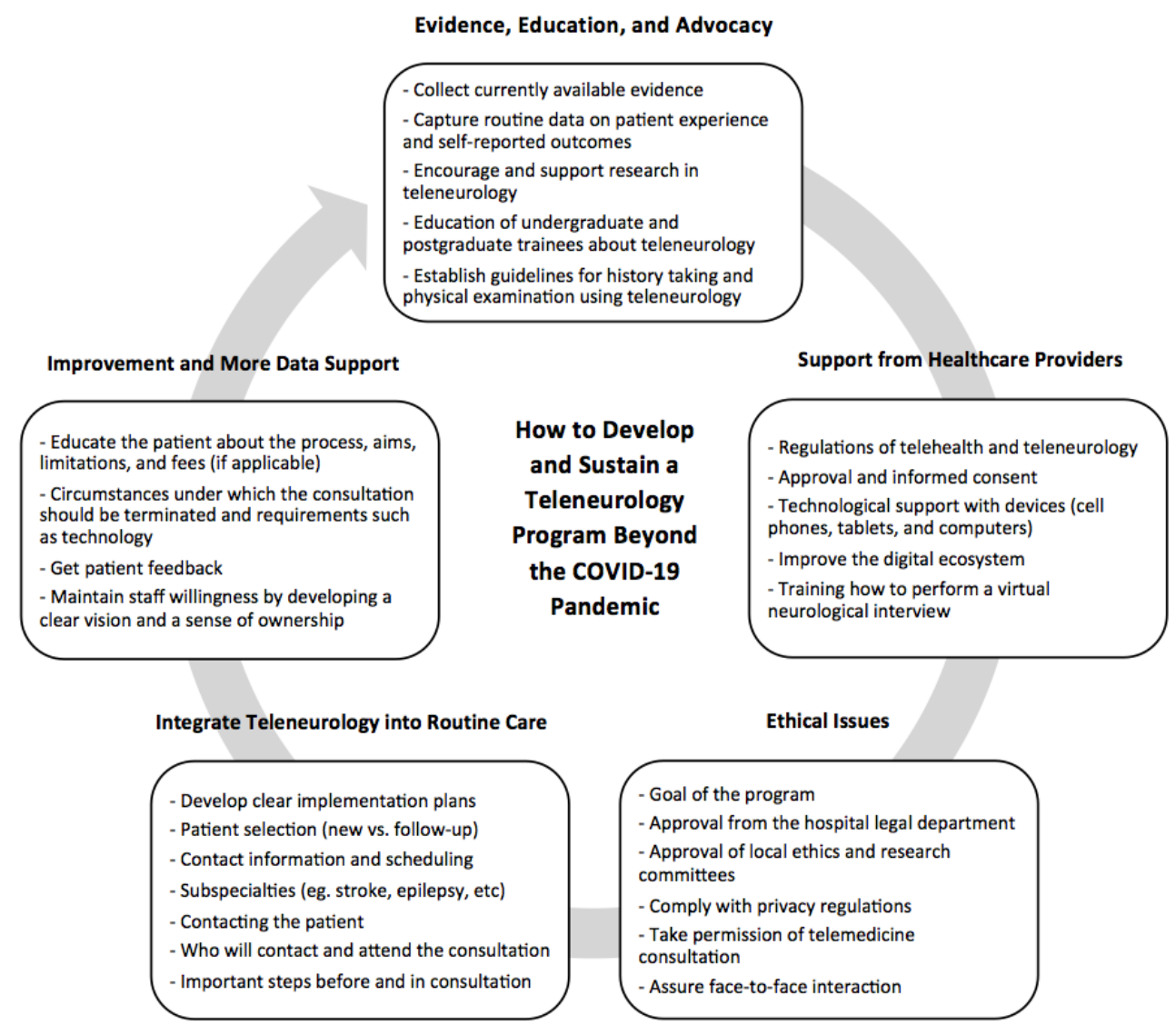

Figure 1 - Schematic representation of how to develop a teleneurology program. ${ }^{1}$

laboratory data, pathological investigation, and all radiological images requested by treating doctors. Although the wide spectrum of telemedicine services was not fully conveyed, contact was maintained with all patients and real-time 2 -way interactive audio visits were conducted. Although no universal consensus exists that high-quality care for neurology patients can be achieved via telemedicine, patients were highly satisfied and the evidence base continues to grow. Synchronous real-time two-way video visits were not implemented at King Abdulaziz Medical City-Jeddah, pending ethical regulations and technical support. However, all computers in virtual neurology clinics are currently equipped with up-to-date and high-capacity largescreen devices with built-in cameras, which make video visits possible once policies and regulations have been established.

Processes and practices for neurology patient care. Mobile devices were used to conduct virtual visits, while electronic medical records were reviewed by neurologists on desktop and laptop computers. Despite the availability of single built-in cameras on desktop and laptop computers and front-and-rear facing cameras on mobile phones, the lack of legal and ethical regulations prohibited video consultations. Suggestions to solve this problem included recorded interviews saved to the electronic medical records, the presence of a second person (e.g., nurse or hospital clerk) during the interview, and obtaining consent to conduct the interview. During the video visit on mobile phones with an epic desktop open simultaneously, rapid medical record review and documentation with great patient satisfaction was enabled. The neurologist was able to discuss the clinical management plan with the patient and order relevant investigations on the spot during the interview. These procedures were performed utilizing voice communications only. Only urgent investigations were ordered, and patients were requested to visit the 
laboratory department directly without attending the clinic, mainly to avoid unnecessary contact with possible COVID-19 patients. Medications were discussed with the patient, ordered on the system, and delivered to the patient at home using courier services. In addition, patients who needed urgent admission were requested to contact the admission office directly after entering the admission request in the system. Thereafter, the admission office allocated a bed to the patient and contacted them by mobile regarding details of hospitalization. Booking of subsequent consultations was performed by a neurologist, and the date was sent to the patient by a mobile text message using the hospital's central telecommunication system. During the pandemic, all scheduled patients in our hospital's electronic system were sent a text message the day before their appointment requesting them not to attend the clinic and informing them that the treating physician will call them on the day of their appointment. Only patients who needed to be evaluated physically were requested to present to the clinic while taking all precautions including washing hands and wearing face masks. Based on clinical statistics, only $2 \%$ of patients visited the clinic for necessary evaluations or to address concerns. In 2012, George et $\mathrm{al}^{7}$ concluded that more than $85 \%$ of the leading United States neurology department use or plan to use telemedicine within the next year, which never happened. This is the same vision and plan that the neurology department at King Abdulaziz Medical City-Jeddah will adopt after the end of the COVID-19 crisis.

Telemedicine and neurologic examination. The essential elements of patient history are well-documented in electronic medical records. Due to the exclusive use of mobile phones for voice communications during virtual clinics, the neurologic examination was not documented. Teleneurology was explored more than 2 decades ago by Craig et al. ${ }^{8}$ The authors conducted a study wherein face-to-face examinations were performed by junior physicians with telemedicine examinations conducted by senior neurology trainees who observed the junior trainees. The gold standard in that study was defined as an in-person examination by a senior neurologist. Investigators found that the telemedicine examination was more sensitive and specific (with the exception of plantar response) than a face-to-face examination. In most studies, teleneurology examination was performed by trained presenters at the patients' location. Although several standardized neurologic rating scales have been validated for teleneurology, there is currently no validated examination for general teleneurology. The American Academy of Neurology and other telemedicine experts provided advice on how to modify common neurological exam techniques to fit the necessary limitations of telemedicine. ${ }^{9}$ During the video face-to-face interview, vital signs were collected, and different components of the nervous system were assessed, including higher cortical functions, cranial nerves, motor, sensory, and coordination examinations. Limitations of telemedicine during neurological examinations include difficulty in conducting a fundoscopic exam, the neuromuscular component, and the vestibular examination. Neurologists should exert more effort to perform neurological examinations in the presence of a relative of the patient (not medical staff) who may utilize the mobile phone and apply the remote instructions given by the neurologist. This has applications for use during a global health crisis such as COVID-19 to limit contact and to reduce the risk of infection. Some elements of the virtual examination can be performed if the patient is alone, while others require an additional person in the home to assist. If the neurologist believes that an in-person visit is needed, and the virtual encounter is inadequate, an appointment can be scheduled with the patient. The need for social distancing while still caring for patients with neurological disorders is considered an urgent call to develop, refine, and validate meaningful teleneurologic examination techniques.

Challenges to successful implementation of telemedicine programs in Saudi Arabia. Although there are few publications on the challenges and difficulties of implementing telemedicine in Saudi Arabia, There are few publications on the challenges and difficulties of implementing telemedicine in Saudi Arabia. In a recent study by Alghamdi et $\mathrm{al}^{10}{ }^{10}$ a short communication was published on the telehealth status in Saudi Arabia during the COVID-19 pandemic. There are major challenges hindering the successful implementation of telemedicine programs and virtual neurology clinics in Saudi Arabia. Some of these challenges relate to the culture and geographic location of Saudi Arabia, and others are independent of the healthcare system. Based on our personal opinion and experience and following a consensus opinion from key stakeholders including patients, families, clinicians, managers, major challenges are listed in Table 1.

Perception and gap of knowledge about telemedicine in Saudi Arabia. According to a few small-scale studies conducted in Saudi Arabia, the implementation of telemedicine in Saudi Arabia was widely accepted with great interest in the technology. ${ }^{11,12}$ However, physicians and medical professionals reported a lack of suitable telemedicine training with several difficulties including patient privacy, high cost of equipment, and lack of cooperation between medical staff and information technologists. In one study, the perception 
of participants at hospitals not adopting telemedicine was higher for all benefits than the perception of participants adopting telemedicine. ${ }^{12}$ This could be related to 2 major factors: high expectations before the actual implementation of telemedicine and inappropriate utilization with a lack of proper training after the implementation of telemedicine. Among the different studies, all participants perceived the benefits of telemedicine in improving the quality of care, enhancing access to healthcare, and saving time and money. Wootton and Bonnardot ${ }^{13}$ examined the benefits of telemedicine in the developing world and concluded that the availability of worldwide satellite communications supported a variety of telemedicine uses for patient care.

Recommendations and future opportunities. The role of teleneurology during the COVID-19 pandemic supported a major shift toward virtual visits which provided value for patients and physicians. ${ }^{14-26} \mathrm{We}$ believe that the government will continue to support telehealth services given the evidence of efficacy, validity, and societal acceptance coupled with the desire of both patients and neurologists to continue practicing virtually. Standardization of the kingdom laws, consent statements, and reimbursement practices will further facilitate the implementation of teleneurology. Once audio and visual clinical data are available in electronic medical records with the help of advanced computing technologies, accurate medical decision-making and superb patient care are expected.

Virtual neurology clinics should be a part of all hospital outpatient departments in Saudi Arabia and be equipped with all essential and basic telemedicine facilities including information and communication technologies in addition to adequate and sustainable financial support. Educational preparation and knowledge dissemination involving patients and their families is mandatory to overcome cultural and societal constraints. These include compatibility with Islamic ethics, human culture, and traditional beliefs. The global COVID-19 pandemic has enabled the rapid introduction of virtual healthcare services in Saudi Arabia with full support from the Saudi government to ensure continuous healthcare provision and to limit exposure and spread of the infection. Figure 1 is demonstrating important steps required to develop a teleneurology program. Undergraduate medical students, residents, and fellows should be trained in teleneurology and telestroke since teleneurology is considered a new subspecialty with fellowship training and courses in several societies and academies including the American Academy of Neurology.
Conclusion. In Saudi Arabia, the COVID-19 pandemic has caused social dislocation, negative economic impact, and a major change in medical practice. Telemedicine has rapidly moved to the frontline of healthcare practice due to the demand for prevention and mitigation strategies. It has been encouraged and facilitated with huge government support. According to the limited currently available literature on telemedicine, there remains an urgent need to improve the perception and knowledge of both medical personnel and patients concerning telemedicine and to move forward with the use of advanced information and communication technology. Although the present study is a singlecenter experience with no statistical data reported, this study is an urgent call for conducting methodological studies tackling different aspects of teleneurology in Saudi Arabia. The experience was new to us, and the implementation was instantaneous, which gave no time to conduct such studies. Future studies should include statistics related to the teleneurology services (actual activity, number of consultations, types of cases, referral outcomes, etc.) as well as evaluation of the perceptions, knowledge, improvements, challenges, and evidence behind teleneurology. Internationally, further work is needed to understand how to sustain telehealth services in the long term and to ensure that these services are available as part of the routine health system.

\section{References}

1. Mahar JH, Rosencrance JG, Rasmussen PA. Telemedicine: Past, present, and future. Cleve Clin J Med 2018; 85: 938-942.

2. Lamay CL. Telemedicine and competitive change in health care. Spine 1997; 22: 88-97.

3. Zhu N, Zhang D, Wang W, Li X, Yang B, Song J, et al. A Novel Coronavirus from Patients with Pneumonia in China, 2019. N Engl J Med 2020; 382: 727-733.

4. Dong E, Du H, Gardner L. An interactive web-based dashboard to track COVID-19 in real time. Lancet Infect Dis 2020; 20: 533-534.

5. Saudi Ministry of Health 2021. [online]. Available at: https:// covid19.moh.gov.sa. [Accessed 13 November 2021].

6. Yezli S, Khan A. COVID-19 social distancing in the Kingdom of Saudi Arabia: Bold measures in the face of political, economic, social and religious challenges. Travel Med Infect Dis 2020; 37: 101692.

7. George BP, Scoglio NJ, Reminick JI, Rajan B, Beck CA, Seidmann A, et al. Telemedicine in Leading US Neurology Departments. Neurohospitalist 2012; 2: 123-128.

8. Craig JJ, Mcconville JP, Patterson VH, Wootton R. Neurological examination is possible using telemedicine. J Telemed Telecare 1999; 5: 177-181.

9. Hatcher-Martin JM, Adams JL, Anderson ER, Bove R, Burrus TM, Chehrenama $M$, et al. Telemedicine in neurology: Telemedicine Work Group of the American Academy of Neurology update. Neurology 2020; 94: 30-38. 
10. Alghamdi SM, Alqahtani JS, Aldhahir AM. Current status of telehealth in Saudi Arabia during COVID-19. J Fam Community Med 2020; 27: 208-211.

11. Althbiti AAJ, Al Khatib FM, AL-Ghalayini NA. Telemedicine: Between Reality and Challenges in Jeddah Hospitals. The Egyptian Journal of Hospital Medicine 2017; 68: 1381-1389.

12. Albarrak AI, Mohammed R, Almarshoud N, Almujalli L, Aljaeed R, Altuwaijiri S, et al. Assessment of physician's knowledge, perception and willingness of telemedicine in Riyadh region, Saudi Arabia. J Infect Public Health 2021; 14: 7-102.

13. Wootton R, Bonnardot L. In what circumstances is telemedicine appropriate in the developing world?. JRSM Short Rep 2010; 1: 37 .

14. Grossman SN, Han SC, Balcer LJ, Kurzweil A, Weinberg H, Galetta SL, et al. Rapid implementation of virtual neurology in response to the COVID-19 pandemic. Neurology 2020; 94: 1077-1087.

15. Smith AC, Thomas E, Snoswell CL, Haydon H, Mehrotra A, Clemensen J, et al. Telehealth for global emergencies: Implications for coronavirus disease 2019 (COVID-19). J Telemed Telecare 2020; 26: 309-313.

16. Thomas EE, Haydon HM, Mehrotra A, Caffery LJ, Snoswell CL, Banbury A, et al. Building on the momentum: Sustaining telehealth beyond COVID-19. J Telemed Telecare 2020; 1357633 X20960638.

17. Patterson V. Teleneurology. J Telemed Telecare 2005; 11: 55-59.

18. Patel UK, Malik P, DeMasi M, Lunagariya A, Jani VB. Multidisciplinary approach and outcomes of tele-neurology: a review. Cureus 2019; 11: e4410.
19. Smith AC, Armfield NR, Coulthard MG, Williams ML, Caffery LJ. Queensland telepaediatric service: a review of the first 15 years of service. Front Digit Health 2020; 2: 587452.

20. Cadilhac DA, Bagot KL, Demaerschalk BM, Hubert G, Schwamm L, Watkins CL, et al. Establishment of an internationally agreed minimum data set for acute telestroke. $J$ Telemed Telecare 2021; 27: 582-589.

21. Al Kasab S, Almallouhi E, Debenham E, Turner N, Simpson $\mathrm{KN}$, Holmstedt CA. Beyond acute stroke: Rate of stroke transfers to a tertiary centre following the implementation of a dedicated inpatient teleneurology network. J Telemed Telecare 2021; 27: 239-243.

22. Bokolo AJ. Use of telemedicine and virtual care for remote treatment in response to covid-19 pandemic. J Med Syst 2020; 44: 132.

23. Hasson SP, Waissengrin B, Shachar E, Hodruj M, Fayngor $\mathrm{R}$, Brezis $\mathrm{M}$, et al. Rapid implementation of telemedicine during the covid-19 pandemic: perspectives and preferences of patients with cancer. Oncologist 2021; 26: e679-e685.

24. Bokolo AJ. Exploring the adoption of telemedicine and virtual software for care of outpatients during and after COVID-19 pandemic. Ir J Med Sci 2021; 190: 1-10.

25. Smith AJ, Pfister BF, Woo EWY, Walters BJ, Blacket B, Page N, et al. Safe and rapid implementation of telemedicine fracture clinics: the impact of the COVID-19 pandemic. ANZ J Surg 2020; 90: 2237-2241.

26. Bokolo AJ. Implications of telehealth and digital care solutions during COVID-19 pandemic: a qualitative literature review. Inform Health Soc Care 2021; 46: 68-83.

\section{Clinical Practice Guidelines}

Clinical Practice Guidelines must include a short abstract. There should be an Introduction section addressing the objective in producing the guideline, what the guideline is about and who will benefit from the guideline. It should describe the population, conditions, health care setting and clinical management/diagnostic test. Authors should adequately describe the methods used to collect and analyze evidence, recommendations and validation. If it is adapted, authors should include the source, how, and why it is adapted? The guidelines should include not more than 50 references, 2-4 illustrations/tables, and an algorithm. 